\title{
A Hamiltonian approach to the planar optimization of mid-course corrections
}

\author{
E. Iorfida ${ }^{1}$ - P. L. Palmer ${ }^{1}$ - M. Roberts R,3 $^{2,3}$
}

Received: 21 August 2015 / Revised: 17 October 2015 / Accepted: 21 November 2015 /

Published online: 22 December 2015

(C) The Author(s) 2015. This article is published with open access at Springerlink.com

\begin{abstract}
Lawden's primer vector theory gives a set of necessary conditions that characterize the optimality of a transfer orbit, defined accordingly to the possibility of adding mid-course corrections. In this paper a novel approach is proposed where, through a polar coordinates transformation, the primer vector components decouple. Furthermore, the case when transfer, departure and arrival orbits are coplanar is analyzed using a Hamiltonian approach. This procedure leads to approximate analytic solutions for the in-plane components of the primer vector. Moreover, the solution for the circular transfer case is proven to be the Hill's solution. The novel procedure reduces the mathematical and computational complexity of the original case study. It is shown that the primer vector is independent of the semi-major axis of the transfer orbit. The case with a fixed transfer trajectory and variable initial and final thrust impulses is studied. The acquired related optimality maps are presented and analyzed and they express the likelihood of a set of trajectories to be optimal. Furthermore, it is presented which kind of requirements have to be fulfilled by a set of departure and arrival orbits to have the same profile of primer vector.
\end{abstract}

Keywords Primer vector - Trajectory optimization · Indirect method · Mid-course corrections · Deep space maneuvers

E. Iorfida

e.iorfida@surrey.ac.uk

P. L. Palmer

p.palmer@surrey.ac.uk

M. Roberts

m.roberts@surrey.ac.uk

1 Surrey Space Centre, University of Surrey, Guildford GU2 7XH, UK

2 Department of Mathematics, University of Surrey, Guildford, UK

3 AIMS Tanzania, PO Box 176, Bagamoyo, Pwani, Tanzania 


\section{Introduction}

The addition of mid-course corrections (or deep space maneuvers, DSM) along an interplanetary transfer has the advantage of decreasing the overall cost of a trajectory. In fact, applying a DSM will modify not only the trajectory but also the boundary conditions (BC) of the orbit (Bernelli-Zazzera et al. 2007). In an impulsive thrust case, this implies modifying the departure and arrival $\Delta \mathbf{V}$ s since the cost of a mission can be defined as sum of impulses (magnitudes of the $\Delta \mathbf{V s}$ ). Generally, this process is done in the scenario of the patched conics method, which means considering a sequence of Keplerian arcs linked together (Battin 1999). The inclusion of a DSM along a transfer orbit has the main drawback of increasing the search space complexity of an optimization problem (Vasile and De Pascale 2006). For this reason, it can be useful to have a method that gives a first guess on where to perturb the orbit for the possible addition of a mid-course correction.

In the early 1960s, Lawden introduced the concept of the primer vector in Lawden (1963). Derived with an indirect optimal control method, this variable is associated with the equation of motion and it has to satisfy a set of necessary conditions (NC) for a transfer to be optimal. In particular if the NCs are not fulfilled, it is energetically favourable to add a DSM, see Conway (2010). For a linear system, Prussing (1995) proved that these conditions are also sufficient.

Later studies such as Lion and Handelsman (1968), Jezewski and Rozendaal (1968), Vinh (1972) and Jezewski (1975) extensively showed where and how to perturb the trajectory for the addition of the correction (with gradient based optimization methods). At present, the primer vector theory is mainly exploited in global optimization scenario as a first estimation of the position of the DSM and therefore it allows mission planners to reduce the size of the search space (Olympio et al. 2006). However, due to its computational complexity, its usage is narrowed also to simple three-body problem cases as in Luo et al. (2010).

The main disadvantage of Lawden's theory is that it is based on solving a boundary value problem (BVP) that involves the state transition matrix (STM) of the motion. Currently, there are no known analytic solutions to the problem and, subsequently, no insights into what determines whether a given transfer will be optimal or not. In papers like Goodyear (1965), a formal solution to the differential equations used to derive the STM is provided. Even if the method presented in Goodyear (1965) is valid for every type of Keplerian transfer, it does not give a complete understanding of the structure of the problem. In fact, it is defined in a general inertial frame and therefore no simplifications in the equations (36 terms expressed as sums and multiplications of series) are possible.

This paper presents a novel approach to the theory which simplifies the structure of the problem. In particular, the case where departure, arrival and transfer orbits are co-planar is analyzed.

\section{Primer vector theory procedure}

The equations of motion can be written as

$$
\dot{\mathbf{x}}(t)=\left[\begin{array}{c}
\dot{\mathbf{r}}(t) \\
\dot{\mathbf{v}}(t)
\end{array}\right]=\left[\begin{array}{c}
\mathbf{v}(t) \\
\mathbf{g}[\mathbf{r}(t)]+\Gamma \mathbf{u}(t)
\end{array}\right]
$$

where $\mathbf{x}(t)$ is the state, $\mathbf{r}(t)$ is the position vector, $\mathbf{v}(t)$ is the velocity vector, $\mathbf{g}(\mathbf{r})$ is the gravitational acceleration, $\mathbf{u}(t)$ represents the unit vector in the direction of the thrust and $\Gamma$ is the impulsive thrust acceleration (Conway 2010). 
As described in Bryson and Ho (1975), there exists a Hamiltonian that defines the system given in Eq. 1:

$$
H=\Gamma+\lambda_{r}^{T} \mathbf{v}(t)+\lambda_{v}{ }^{T}[\mathbf{g}(\mathbf{r})+\Gamma \mathbf{u}],
$$

where $\lambda_{r / v}$ are the co-state variables associated, respectively, to the position and the velocity of Eq. 1. In a classic optimization problem, the cost function can be defined as the total propellant consumption. For an impulsive thrust case, the cost is simply the sum of the magnitudes of the $N$ impulses fired along the transfer arc (Conway 2010)

$$
J=\sum_{i=1}^{N} \Delta V_{i}
$$

The adjoint equations referred to Eq. 2 which minimize the cost are

$$
\begin{aligned}
& {\dot{\lambda_{r}}}^{\mathrm{T}}=-\frac{\partial H}{\partial \mathbf{r}}=-\lambda_{v} \mathbf{G}(\mathbf{r}) \\
& {\dot{\lambda_{v}}}^{\mathrm{T}}=-\frac{\partial H}{\partial \mathbf{v}}=-\boldsymbol{\lambda}_{r}{ }^{\mathrm{T}}
\end{aligned}
$$

where $\mathbf{G}(\mathbf{r}) \triangleq \frac{\partial \mathbf{g}(\mathbf{r})}{\partial \mathbf{r}}$ is the $3 \times 3$ gravity gradient matrix.

The primer vector $\mathbf{p}(t)$ and its derivative $\dot{\mathbf{p}}(t)$ have been defined in Lawden (1963) as

$$
\begin{aligned}
& \mathbf{p}(t) \triangleq-\lambda_{v}(t) \\
& \dot{\mathbf{p}}(t)=\lambda_{r}(t) .
\end{aligned}
$$

Equations 4, 5, 6 and 7 combined together, give the 'primer vector equation':

$$
\ddot{\mathbf{p}}=\mathbf{G}(\mathbf{r}) \mathbf{p} \text {. }
$$

Furthermore, Lawden characterized the theory with a set of NC for the impulsive thrust case, when the cost is simply expressed as a sum of the magnitudes of $\Delta \mathbf{V s}$ (as in Eq. 3). The satisfaction of the NCs defines the optimality of a trajectory [extensive explanation in Jezewski (1975)]:

1. The primer vector and its derivative are continuous everywhere.

2. The magnitude of the primer vector is always below 1 , aside from the instants where the impulse occurs, where $p=1$.

3. At the impulse times, the primer vector is a unit vector in the direction of the thrust.

4. As a consequence of the above conditions, $d p / d t=\dot{p}=\dot{\mathbf{p}}^{T} \mathbf{p}=0$ at an intermediate impulse.

Equation 7 needs to be integrated to obtain the primer vector profile along the transfer orbit and then to verify if the $\mathrm{NC}$ are satisfied. Its solution can be expressed in the form of

$$
\left[\begin{array}{c}
\mathbf{p}(t) \\
\dot{\mathbf{p}}(t)
\end{array}\right]=\boldsymbol{\Phi}\left(t, t_{0}\right)\left[\begin{array}{l}
\mathbf{p}\left(t_{0}\right) \\
\dot{\mathbf{p}}\left(t_{0}\right)
\end{array}\right]
$$

where $\boldsymbol{\Phi}$ is the STM associated to the state (Jezewski 1975). The third NC gives the BC on the primer vector, defining it as unit vector in the direction of the thrust:

$$
\begin{gathered}
\mathbf{p}_{0} \equiv \mathbf{p}\left(t_{0}\right)=\frac{\Delta \mathbf{V}_{0}}{\left\|\Delta \mathbf{V}_{0}\right\|} \\
\mathbf{p}_{f} \equiv \mathbf{p}\left(t_{f}\right)=\frac{\Delta \mathbf{V}_{f}}{\left\|\Delta \mathbf{V}_{f}\right\|} .
\end{gathered}
$$


However, both initial conditions on $\mathbf{p}(t)$ and $\dot{\mathbf{p}}(t)$ are required for the propagation of Eq. 9 . $\mathbf{p}_{0}$ is known from Eq. 10 and the initial condition of $\dot{\mathbf{p}}$ comes inverting Eq. 9 as:

$$
\dot{\mathbf{p}}\left(t_{0}\right)=\boldsymbol{\Phi}_{12}^{-1}\left(t_{f}, t_{0}\right) \cdot\left[\mathbf{p}\left(t_{f}\right)-\boldsymbol{\Phi}_{11}\left(t_{f}, t_{0}\right) \cdot \mathbf{p}\left(t_{0}\right)\right]
$$

where $\boldsymbol{\Phi}_{11}$ and $\boldsymbol{\Phi}_{12}$ represent partition matrices of $\boldsymbol{\Phi}$, and they are the only unknowns of Eq. 11. As a consequence, this procedure implies solving a BVP where firstly the STM is integrated along the arc to solve Eq. 11 for $\dot{\mathbf{p}}\left(t_{0}\right)$ and, afterwards, Eq. 9 is propagated to verify Lawden's NCs.

\section{Polar coordinates transformation}

This paper presents a novel procedure framed in a local polar coordinate system defined by $\hat{\mathbf{e}}_{r}, \hat{\mathbf{e}}_{\vartheta}$ and $\hat{\mathbf{e}}_{h}$. They are, respectively, unit vectors in the direction of the local position vector, of the derivative of $\hat{\mathbf{e}}_{r}$ with respect to the true anomaly $(v)$ and of the orbital momentum vector $(\mathbf{h})$ :

$$
\hat{\mathbf{e}}_{r}=\frac{\mathbf{r}}{\|\mathbf{r}\|} ; \quad \hat{\mathbf{e}}_{\vartheta}=-\hat{\mathbf{e}}_{r} \times \hat{\mathbf{e}}_{h} ; \quad \hat{\mathbf{e}}_{h}=\frac{\mathbf{h}}{\|\mathbf{h}\|} .
$$

It can be demonstrated that this coordinate system is an eigenvector basis of the gravity gradient matrix $\mathbf{G}$ (Eq. 8), defined to first order approximation as

$$
\mathbf{G}=-\frac{\mu}{r^{3}} \mathbf{I}_{3}+\frac{3 \mu}{r^{5}} \mathbf{r} \cdot \mathbf{r}^{T}
$$

The corresponding eigenvalues of $\mathbf{G}$, with respect to the unit vectors of Eq. 12, are $2 \mu / r^{3}$, of algebraic multiplicity 1 , and $-\mu / r^{3}$, of algebraic multiplicity 2 .

Combining together Eq. 8 and the eigen-decomposition of $\mathbf{G}$, the primer vector equation can be expressed in polar form as

$$
\ddot{\mathbf{p}}=\mathbf{G p}=\frac{\mu}{r^{3}}\left(2 p_{r} \hat{\mathbf{e}}_{r}-p_{\vartheta} \hat{\mathbf{e}}_{\vartheta}-p_{h} \hat{\mathbf{e}}_{h}\right) .
$$

Finally the first derivative of the primer vector with respect to time in polar coordinates can be derived:

$$
\frac{d \mathbf{p}}{d t}=\underbrace{\left(\dot{p}_{r}-\frac{h}{r^{2}} p_{\vartheta}\right) \hat{\mathbf{e}}_{r}+\left(\dot{p}_{\vartheta}+\frac{h}{r^{2}} p_{r}\right) \hat{\mathbf{e}}_{\vartheta}}_{\text {IN PLANE }}+\underbrace{\dot{p}_{h} \hat{\mathbf{e}}_{h}}_{\text {OUT OF PLANE }} .
$$

From Eq. 15, it is clear that the out-of-plane component is decoupled from the in-plane components.

The analysis related to the out-of-plane component is treated in a complementary paper from the authors, now in press.

\subsection{In-plane components}

Concerning the in-plane part of Eq. 15, $p_{r}$ and $p_{\vartheta}$ do not have formal analytic solutions. Even so, it is possible to find an analytic approximation for which the time-variant variable is considered to be constant for a small integration step size. 
From Eq. 15, two new variables can be defined:

$$
\left\{\begin{array}{l}
P_{r} \triangleq \dot{p}_{r}-\frac{h}{r^{2}} p_{\vartheta} \\
P_{\vartheta} \triangleq \dot{p}_{\vartheta}+\frac{h}{r^{2}} p_{r}
\end{array} .\right.
$$

$P_{r}$ and $P_{\vartheta}$ are the components of the first derivative of the primer vector with respect to time in the local orbital reference frame. Therefore, the in-plane part of Eq. 15 becomes

$$
\dot{\mathbf{p}}=P_{r} \hat{\mathbf{e}}_{r}+P_{\vartheta} \hat{\mathbf{e}}_{\vartheta} .
$$

Taking into account the derivatives with respect to time of the eigenvectors:

$$
\left\{\begin{array}{l}
\dot{\hat{\mathbf{e}}}_{r}=\dot{v} \hat{\mathbf{e}}_{\vartheta} \\
\dot{\hat{\mathbf{e}}}_{\vartheta}=-\dot{v} \hat{\mathbf{e}}_{r}, \\
\dot{\hat{\mathbf{e}}}_{h}=0
\end{array},\right.
$$

the second derivative of the primer vector can be easily derived as

$$
\ddot{\mathbf{p}}=\left(\dot{P}_{r}-\frac{h}{r^{2}} P_{\vartheta}\right) \hat{\mathbf{e}}_{r}+\left(\dot{P}_{\vartheta}+\frac{h}{r^{2}} P_{r}\right) \hat{\mathbf{e}}_{\vartheta} .
$$

The primer vector equation in polar form (Eq. 14) combined with Eq. 16 and Eq. 19 gives the derivatives of $P_{r}$ and $P_{\vartheta}$

$$
\left\{\begin{array}{l}
\dot{P}_{r}=\frac{2 \mu}{r^{3}} p_{r}+\frac{h}{r^{2}} P_{\vartheta} \\
\dot{P}_{\vartheta}=-\frac{\mu}{r^{3}} p_{\vartheta}-\frac{h}{r^{2}} P_{r}
\end{array} .\right.
$$

To simplify the structure of the equations, two new variables can be defined:

$$
\begin{aligned}
& \sigma \triangleq \frac{r}{l} \equiv \frac{1}{1+e \cos v}, \\
& \omega \triangleq \frac{h}{r^{2}}=\frac{h}{(l \sigma)^{2}}
\end{aligned},
$$

which satisfy also the following equivalence

$$
\sigma \cdot \omega^{2}=\frac{r}{l} \frac{h^{2}}{r^{4}}=\frac{\mu}{r^{3}} \frac{r}{r} \frac{l}{l}=\frac{\mu}{r^{3}}
$$

where $l=a\left(1-e^{2}\right)$ is the semi-latus rectum of the orbit (Battin 1999).

The new variables, linked together, give a new first order ODE set in $\left[p_{r}, p_{\vartheta}, P_{r}, P_{\vartheta}\right]$ :

$$
\left[\begin{array}{c}
\dot{p}_{r} \\
\dot{p}_{\vartheta} \\
\dot{P}_{r} \\
\dot{P}_{\vartheta}
\end{array}\right]=\left[\begin{array}{cccc}
0 & \omega & 1 & 0 \\
-\omega & 0 & 0 & 1 \\
2 \sigma \omega^{2} & 0 & 0 & \omega \\
0 & -\sigma \omega^{2} & -\omega & 0
\end{array}\right]\left[\begin{array}{c}
p_{r} \\
p_{\vartheta} \\
P_{r} \\
P_{\vartheta}
\end{array}\right] .
$$

Equation 23 can be represented in compact matrix format as

$$
\dot{\mathbf{p}}_{\text {polar }}(t)=\mathbf{M}(t) \mathbf{p}_{\text {polar }}(t) \text {. }
$$




\subsection{Palmer coordinates conversion and integration scheme}

The system of Eq. 23 can be written in terms of the Hamiltonian:

$$
H_{\mathrm{PV}}=\frac{1}{2}\left(P_{r}^{2}+P_{\vartheta}^{2}\right)+\omega\left(p_{\vartheta} P_{r}-p_{r} P_{\vartheta}\right)-\frac{1}{2} \sigma \omega^{2}\left(2 p_{r}{ }^{2}-p_{\vartheta}{ }^{2}\right) .
$$

If $H_{\mathrm{PV}}$ is compared to the one of Hill's problem, $H_{\mathrm{Hill}}$ of Quinn et al. (2010), there is a noticeable similarity:

$$
H_{\text {Hill }}=\frac{1}{2}\left(p_{x}^{2}+p_{y}{ }^{2}\right)+\Omega\left(y p_{x}-x p_{y}\right)-\frac{1}{2} \Omega^{2}\left(2 x^{2}-y^{2}\right) .
$$

The substantial difference between the two Hamiltonian forms is that, while in $H_{\text {Hill }}$ the coefficient $\Omega$ is constant during the motion, in $H_{\mathrm{PV}}$ both $\sigma$ and $\omega$ vary in time. However, for the circular orbit case (Hill's problem), $\sigma=1$ and $\omega=\Omega$, which implies a one-to-one correspondence between the two Hamiltonians. This fact validates a fundamental result: for a circular orbit transfer there is an analytic solution to the planar primer vector problem, which is exactly the Hill's solution.

For Hill's problem, the motion of the in-plane state vector of Eq. $26, \mathbf{z}=(\Omega x, \dot{x}, \Omega y, \dot{y})^{\mathrm{T}}$, can be separated into two parts: an oscillation and a linear drift. This result is presented in Palmer (2007), where the transformation of coordinates $\tilde{\mathbf{p}}=\mathcal{P} \mathbf{z}=\left(p_{1}, p_{2}, p_{3}, p_{4}\right)^{T}$ on $\mathbf{z}$ allows this separation to be explicit (as a note, the original notation of Palmer (2007) has been changed to avoid confusion).

The combination of Eqs. 16, 25 and 26 results in the relationship between the state vector $\mathbf{z}$ and $\mathbf{p}_{\text {polar }}$ :

$$
\left\{\begin{array}{l}
x=p_{r} \\
y=p_{\vartheta}
\end{array} ;\left\{\begin{array} { l } 
{ p _ { x } = P _ { r } = \dot { x } - \Omega y } \\
{ p _ { y } = P _ { \vartheta } = \dot { y } + \Omega x }
\end{array} \Rightarrow \left\{\begin{array}{l}
\dot{x}=p_{x}+\Omega y=P_{r}+\omega p_{\vartheta} \\
\dot{y}=p_{y}-\Omega x=P_{\vartheta}-\omega p_{r}
\end{array}\right.\right.\right.
$$

where the frequency associated with $\mathbf{z}$ is $\Omega$, while the one of $\mathbf{p}_{\text {polar }}$ is $\omega$.

If the transformation of coordinates presented in Palmer (2007) is applied to the primer vector problem (Eq. 27), it becomes

$$
\left[\begin{array}{l}
p_{1} \\
p_{2} \\
p_{3} \\
p_{4}
\end{array}\right]=\left[\begin{array}{cccc}
-3 & 0 & 0 & -2 \\
0 & 1 & 0 & 0 \\
0 & 2 & -1 & 0 \\
2 & 0 & 0 & 1
\end{array}\right]\left[\begin{array}{c}
\omega p_{r} \\
P_{r}+\omega p_{\vartheta} \\
\omega p_{\vartheta} \\
P_{\vartheta}-\omega p_{r}
\end{array}\right]
$$

\subsubsection{Integration of the Palmer coordinates}

The coordinates $\tilde{\mathbf{p}}$ (also called Palmer coordinates) can be expressed as functions of $\left(p_{r}, p_{\vartheta}, P_{r}, P_{\vartheta}\right)$ as shown in Eq. 28 , where two couplings are evident: one between $p_{1}$ and $p_{4}$ and the other between $p_{2}$ and $p_{3}$.

If $\tilde{\mathbf{p}}$ is first combined with the system of Eq. 23 and then differentiated with respect to time (while $\sigma$, and therefore $\omega$, are kept constant for small intervals of time), it yields

$$
\left[\begin{array}{l}
\dot{p}_{1} \\
\dot{p}_{2} \\
\dot{p}_{3} \\
\dot{p}_{4}
\end{array}\right]=\omega\left[\begin{array}{cccc}
0 & 4 \sigma-3 & -2(\sigma-1) & 0 \\
2 \sigma-3 & 0 & 0 & 4(\sigma-1) \\
4(\sigma-1) & 0 & 0 & 8 \sigma-5 \\
0 & -2(\sigma-1) & \sigma-1 & 0
\end{array}\right]\left[\begin{array}{l}
p_{1} \\
p_{2} \\
p_{3} \\
p_{4}
\end{array}\right]
$$


Fig. 1 Elliptical transfer orbit divided into three regions (I, II, III) according to the nature of the eigenvalues $\lambda_{1,2} \pm$

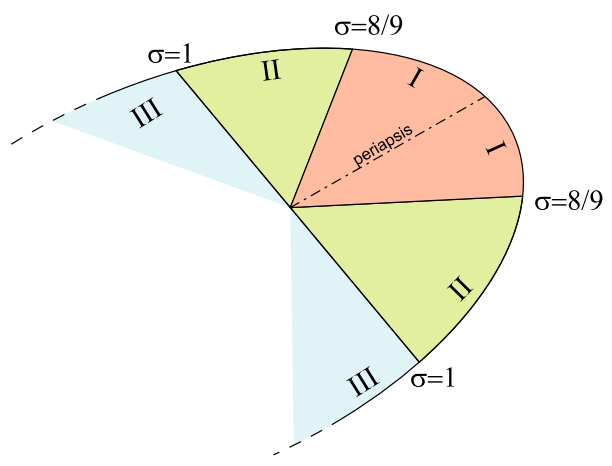

From this system of equations, it is then possible to differentiate even further to a second order system as

$$
\left[\begin{array}{l}
\ddot{p}_{1} \\
\ddot{p}_{2} \\
\ddot{p}_{3} \\
\ddot{p}_{4}
\end{array}\right]=\omega^{2}\left[\begin{array}{cccc}
-(2 \sigma-1) & 0 & 0 & -2(\sigma-1) \\
0 & -(2 \sigma-1) & 2(\sigma-1) & 0 \\
0 & -2(\sigma-1) & 3(\sigma-1) & 0 \\
2(\sigma-1) & 0 & 0 & 3(\sigma-1)
\end{array}\right]\left[\begin{array}{l}
p_{1} \\
p_{2} \\
p_{3} \\
p_{4}
\end{array}\right] .
$$

In Eq. 30, the variables are coupled again ( $p_{1}$ with $p_{4}$ and $p_{2}$ with $p_{3}$ ).

The structure of the problem allows to have a 4 th order ODE in $p_{1}$ in the form of

$$
p_{1}{ }^{\mathrm{IV}}-\omega^{2}(\sigma-2) p_{1}{ }^{\mathrm{II}}-\omega^{4}(\sigma-1)(2 \sigma+1)=0 .
$$

Assuming that the coefficients of Eq. 31 are constant for a small interval of time, the solution will be $p_{1} \approx e^{\omega \lambda t}$.

The characteristic equation in $\lambda$ of Eq. 31 is

$$
\lambda^{4}-\omega^{2}(\sigma-2) \lambda^{2}-\omega^{4}(\sigma-1)(2 \sigma+1)=0,
$$

whose solutions are

$$
\begin{aligned}
& \lambda_{1}^{ \pm}=\frac{\sqrt{2}}{2 \omega} \sqrt{(\sigma-2) \pm \sqrt{9 \sigma^{2}-8 \sigma}} \\
& \lambda_{2}^{ \pm}=-\frac{\sqrt{2}}{2 \omega} \sqrt{(\sigma-2) \pm \sqrt{9 \sigma^{2}-8 \sigma}} .
\end{aligned}
$$

The nature of $\lambda_{1,2}{ }^{ \pm}$depends upon the value of $\sigma$. If $\Delta_{\sigma} \triangleq 9 \sigma^{2}-8 \sigma$, there are three cases (the interval $\sigma<0$ is not taken into consideration because it does not have any physical meaning):

- $\boldsymbol{\Delta}_{\sigma}>\mathbf{0}: \sigma>8 / 9 \cdot \lambda_{1,2^{ \pm}}$can be either real (if $\operatorname{sign}\left((\sigma-2) \pm \Delta_{\sigma}\right)>0$ ) or imaginary (if $\operatorname{sign}\left((\sigma-2) \pm \Delta_{\sigma}\right)<0$ );

- $\boldsymbol{\Delta}_{\boldsymbol{\sigma}}=\mathbf{0}$ : $\sigma$ can be either 0 or $8 / 9$ and $\lambda_{1,2}{ }^{ \pm}$are always imaginary;

- $\boldsymbol{\Delta}_{\sigma}<\mathbf{0}: 0<\sigma<8 / 9$ and $\lambda_{1,2}{ }^{ \pm}$are generally complex.

In the case of an elliptic orbit the nature of the eigenvalues can be summarized, as in Fig. 1, into three distinguished areas:

Area (I) $0<\sigma<8 / 9$ : corresponds to the case of $\Delta_{\sigma}<0$, and includes the periapsis of the transfer orbit, which is the point where $\sigma$ has its minimum value, $\sigma_{\min }=(1+e)^{-1}$. In this interval the eigenvalues are complex. 
Area (II) $8 / 9 \leq \sigma<1$ : corresponds to the case of $\Delta_{\sigma}>0$ with $\operatorname{sign}\left((\sigma-2) \pm \Delta_{\sigma}\right)<0$, and the eigenvalues are imaginary. For $\sigma=1$, that is $r=l, \lambda_{1,2}+=0$ while $\lambda_{1,2^{-}}$are imaginary.

Area (III) $\sigma>1$ : corresponds to the case of $\Delta_{\sigma}>0 ; \lambda_{1,2}{ }^{+}$are real, while $\lambda_{1,2}{ }^{-}$are imaginary. At the apoapsis (included in this area) $\sigma$ reaches its maximum value $\sigma_{\max }=(1-e)^{-1}$.

For values of the eccentricity smaller than $1 / 8, \Delta \geq 0$ and $\sigma \geq 8 / 9$, therefore there is no Area (I). In other words, the eigenvalues are either purely imaginary or real (Area (II) and Area (III)).

After the aforementioned considerations, the complete solution of Eq. 31 can be derived, and it simply is

$$
p_{1}=A e^{\omega \lambda_{1}^{+} t}+B e^{\omega \lambda_{2}^{+} t}+C e^{\omega \lambda_{1}^{-} t}+D e^{\omega \lambda_{2}^{-} t}
$$

where $\mathbf{A}=(A, B, C, D)^{\mathrm{T}}$ is a vector of coefficients given by the initial conditions of $p_{1}$.

The other components of $\tilde{\mathbf{p}}$ can be evaluated as for $p_{1}$, resulting in a formal matrix expression

$$
\tilde{\mathbf{p}}=\boldsymbol{\Sigma}(\sigma)^{-1} \tilde{\mathbf{E}}(t, \sigma)^{-1} \mathbf{A} .
$$

$\tilde{\mathbf{E}}(t, \sigma)$ is a diagonal matrix with independent exponential functions as elements of the diagonal, functions of time $t$ and $\omega(\sigma)$ :

$$
\tilde{\mathbf{E}}=\left[\begin{array}{cccc}
e^{\omega \lambda_{2}^{+} t} & 0 & 0 & 0 \\
0 & e^{\omega \lambda_{1}^{+} t} & 0 & 0 \\
0 & 0 & e^{\omega \lambda_{2}^{-} t} & 0 \\
0 & 0 & 0 & e^{\omega \lambda_{1}^{-} t}
\end{array}\right]
$$

while $\boldsymbol{\Sigma}(\sigma)$ is a polynomial matrix in $\sigma$, written as

$$
\Sigma=\frac{(\sigma-1)}{(2 \sigma+1) \sqrt{\Delta_{\sigma}}}\left[\begin{array}{cccc}
(2 \sigma+1) \xi^{-} & -\lambda_{1}^{+} \gamma^{-} & \lambda_{1}^{+} \delta^{-} & -(2 \sigma+1) \\
(2 \sigma+1) \xi^{-} & \lambda_{1}^{+} \gamma^{-} & -\lambda_{1}^{+} \delta^{-} & -(2 \sigma+1) \\
-(2 \sigma+1) \xi^{+} & \lambda_{1}^{-} \gamma^{+} & -\lambda_{1}^{-} \delta^{+} & (2 \sigma+1) \\
-(2 \sigma+1) \xi^{+} & -\lambda_{1}^{-} \gamma^{+} & \lambda_{1}^{-} \delta^{+} & (2 \sigma+1)
\end{array}\right]
$$

The parameters $\xi^{ \pm}, \gamma^{ \pm}$and $\delta^{ \pm}$are also only functions of $\sigma$ and they are defined as

$$
\left\{\begin{array}{l}
\delta^{ \pm} \triangleq\left[(-3 \sigma+1) \mp \sqrt{\Delta_{\sigma}}\right] \\
\gamma^{ \pm} \triangleq \frac{1}{4(1-\sigma)}\left[\left(24 \sigma^{2}-25 \sigma+4\right) \pm(8 \sigma-5) \sqrt{\Delta_{\sigma}}\right] \\
\xi^{ \pm \triangleq} \triangleq \frac{\left.(5 \sigma-4) \pm \sqrt{\Delta_{\sigma}}\right)}{4(1-\sigma)}
\end{array} .\right.
$$

\subsubsection{Conversion from time to true anomaly}

The propagation process presented so far, is based on time as the integration variable. In orbital dynamics it is sometimes more convenient to use true anomaly rather than time; therefore, the variable conversion is

$$
\frac{d v}{d t}=\omega \Rightarrow \frac{d v}{\omega}=d t \Rightarrow \frac{v}{\omega}=t
$$

Since both $\sigma$ and $\omega$ have been considered constant along an integration step, the conversion from time to true anomaly does not affect the solutions of Eq. 35, a part from a factor $\omega$. 
Fig. 2 Step Propagation scheme of $v$ and $\sigma$, with $\sigma=$ const along every interval of $v$. A general $k$ th state is highlighted with the two limiting values $\left(k^{ \pm}\right)$in red

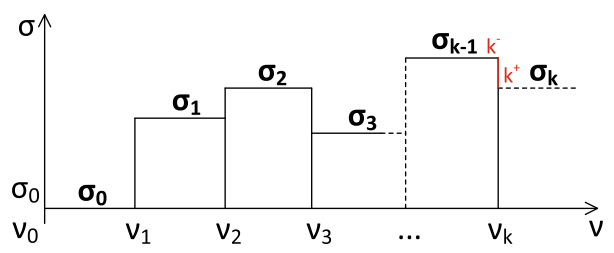

The ODE of Eq. 29 can be converted into true anomaly as

$$
\tilde{\mathbf{p}}^{\prime}=\left[\begin{array}{cccc}
0 & 4 \sigma-3 & -2(\sigma-1) & 0 \\
2 \sigma-3 & 0 & 0 & 4(\sigma-1) \\
4(\sigma-1) & 0 & 0 & 8 \sigma-5 \\
0 & -2(\sigma-1) & \sigma-1 & 0
\end{array}\right] \tilde{\mathbf{p}},
$$

where the symbol $\left({ }^{\prime}\right)$ represents the derivative with respect to $v$, and in compact form it is equivalent to

$$
\tilde{\mathbf{p}}^{\prime}=\Lambda \tilde{\mathbf{p}}
$$

This implies that the solution shown in Eq. 35 remains in the same form:

$$
\mathbf{A}=\mathbf{E}(v, \sigma) \boldsymbol{\Sigma}(\sigma) \tilde{\mathbf{p}}
$$

The only difference appears in $\mathbf{E}$, as

$$
\mathbf{E}(v, \sigma)=\left[\begin{array}{cccc}
e^{\lambda_{2}^{+} v} & 0 & 0 & 0 \\
0 & e^{\lambda_{1}^{+} v} & 0 & 0 \\
0 & 0 & e^{\lambda_{2}^{-} v} & 0 \\
0 & 0 & 0 & e^{\lambda_{1}^{-} v}
\end{array}\right]
$$

where the exponents are explicit functions in $v$. Through the transformation from time to true anomaly, the importance of the Palmer coordinates is even more clear. In fact, using $\tilde{\mathbf{p}}$ allows a solution which is a linear combination of the eigenvalues of the matrix $\mathbf{M}$ (Eq. 24). This solution depends only on $\sigma$ through the polynomial matrix $\boldsymbol{\Sigma}$ and the diagonal matrix $\mathbf{E}$, with an explicit propagation variable $v$.

\subsubsection{Matrices propagation scheme}

Since Eq. 42 assumes that $\sigma$ is constant for small interval of $v$, it is required to apply a 'double' propagation for the Palmer coordinates. This means that every time that the true anomaly is propagated to the next step, the corresponding value of $\sigma$ must be updated, as shown in the scheme of Fig. 2.

At the initial time the coefficients depend upon the initial value of the Palmer coordinates as

$$
\mathbf{A}_{0}=\mathbf{E}\left(v_{0}, \sigma_{0}\right) \boldsymbol{\Sigma}\left(\sigma_{0}\right) \tilde{\mathbf{p}}_{0} .
$$

Knowing $\mathbf{A}_{0}, \tilde{\mathbf{p}}$ can be propagated to the step '1', but only just before the update of $\sigma$. In fact the value of $\tilde{\mathbf{p}}$ after the update will be different. To have a formal expression, this condition can be considered at a generic $k$ th propagation state (see Fig. 2), where the values of the Palmer coordinates just before $\left(\tilde{\mathbf{p}}_{k^{-}}\right)$and after $\left(\tilde{\mathbf{p}}_{k^{+}}\right)$the variation of $\sigma$ are

$$
\begin{aligned}
\tilde{\mathbf{p}}_{k^{-}} & =\boldsymbol{\Sigma}^{-1}\left(\sigma_{k-1}\right) \mathbf{E}^{-1}\left(v_{k}, \sigma_{k-1}\right) \mathbf{A}_{k-1} \\
\tilde{\mathbf{p}}_{k^{+}} & =\boldsymbol{\Sigma}^{-1}\left(\sigma_{k}\right) \mathbf{E}^{-1}\left(v_{k}, \sigma_{k}\right) \mathbf{A}_{k}
\end{aligned}
$$


In the limit towards $k, \tilde{\mathbf{p}}_{k^{ \pm}}$are not the same because they depend on two different $\sigma \mathrm{s}$. However, for the primer vector the continuity must be guaranteed along the whole transfer arc. For this reason, a simple propagation scheme (Fig. 2) has to be used, instead of a more complex one (e.g. leapfrog method). In fact, it guarantees that a 'continuity check' on the primer vector is executed at every update of $\sigma$.

From Eq. 28 a relationship between $\mathbf{p}_{\text {polar }}$ and $\tilde{\mathbf{p}}$ can be derived

$$
\tilde{\mathbf{p}}=\left[\begin{array}{cccc}
-\omega & 0 & 0 & -2 \\
0 & \omega & 1 & 0 \\
0 & \omega & 2 & 0 \\
\omega & 0 & 0 & 1
\end{array}\right] \mathbf{p}_{\text {polar }} \equiv \mathbf{K}(\omega) \mathbf{p}_{\text {polar }}
$$

The matrix $\mathbf{K}(\omega)$, depending on $\omega$, will be different before and after the update. Subsequently, the relations between the Palmer coordinate and the primer vector at a generic $k$ th step are

$$
\begin{aligned}
\mathbf{p}_{\text {polar }_{k}} & =\mathbf{K}^{-1}\left(\omega_{k-1}\right) \tilde{\mathbf{p}}_{k^{-}} \\
\tilde{\mathbf{p}}_{k^{+}} & =\mathbf{K}\left(\omega_{k}\right) \mathbf{p}_{\text {polar }_{k}}
\end{aligned}
$$

Combining Eqs. 48 and 49 gives the matrix which guarantees the continuity of the primer vector, defined as $\mathcal{I}_{\omega_{k}}$ :

$$
\tilde{\mathbf{p}}_{k^{+}}=\mathbf{K}\left(\omega_{k}\right) \mathbf{K}^{-1}\left(\omega_{k-1}\right) \tilde{\mathbf{p}}_{k^{-}} \triangleq \mathcal{I}_{\omega_{k}} \tilde{\mathbf{p}}_{k^{-}}
$$

The procedure for the propagation of the Palmer coordinates from its initial conditions $\tilde{\mathbf{p}}_{0}$ to a generic $k$ th state can be then formalized as

$$
\tilde{\mathbf{p}}_{k}=\left(\prod_{i=0}^{k-1} \mathcal{I}_{\omega_{i}} \boldsymbol{\Sigma}^{-1}\left(\sigma_{i}\right) \mathbf{E}^{-1}\left(v_{i+1}, \sigma_{i}\right) \mathbf{E}\left(v_{i}, \sigma_{i}\right) \boldsymbol{\Sigma}\left(\sigma_{i}\right)\right) \tilde{\mathbf{p}}_{0} .
$$

In summary, Eq. 51 can be written in matrix form as

$$
\tilde{\mathbf{p}}_{k}=\boldsymbol{\Psi}\left(v_{k}, v_{0}\right) \tilde{\mathbf{p}}_{0} .
$$

where $\boldsymbol{\Psi}\left(v_{k}, v_{0}\right)$ represents the propagation matrix of the Palmer coordinates.

The final step of the scheme is to convert the Palmer coordinates back into primer vector at the BC. Combining the definitions of $P_{r}$ and $P_{\vartheta}$ (Eq. 16) and Eq. 28, there is (where $\mathbf{p}_{t o t}$ clearly refers to the the primer vector and its derivative with respect to time):

$$
\tilde{\mathbf{p}}=\left[\begin{array}{cccc}
-3 \omega & 0 & 0 & -2 \\
0 & 0 & 1 & 0 \\
0 & -\omega & 2 & 0 \\
2 \omega & 0 & 0 & 1
\end{array}\right]\left[\begin{array}{c}
p_{r} \\
p_{\vartheta} \\
\dot{p}_{r} \\
\dot{p}_{\vartheta}
\end{array}\right] \equiv \mathbf{B}(\omega) \mathbf{p}_{t o t}
$$

Equations 52 and 53 together yield to

$$
\mathbf{p}_{t o t f}=\mathbf{B}^{-1}\left(\omega_{f}\right) \boldsymbol{\Psi}\left(v_{f}, v_{0}\right) \mathbf{B}\left(\omega_{0}\right) \mathbf{p}_{\text {tot } 0} \triangleq \tilde{\boldsymbol{\Phi}}_{(f, 0)} \mathbf{p}_{\text {tot } 0}
$$

where $\tilde{\boldsymbol{\Phi}}_{(f, 0)}$ has been defined as the total propagation matrix of the primer vector.

Equation 54 summarizes the whole propagation scheme for the primer vector. 


\subsubsection{Independence from the semi-major axis of the transfer orbit}

The value of the 'continuity' matrix, $\mathcal{I}_{\omega}$, defined in Eq. 50, is actually independent from the explicit value of $\omega$ (and therefore from $a$ ) and it is equal to

$$
\mathcal{I}_{\omega_{k}}=\left[\begin{array}{cccc}
\left(\omega_{k} / \omega_{k-1}+2\right) & 0 & 0 & \left(-2 \omega_{k} / \omega_{k-1}+2\right) \\
0 & \left(2 \omega_{k} / \omega_{k-1}-1\right) & \left(-\omega_{k} / \omega_{k-1}+1\right) & 0 \\
0 & \left(2 \omega_{k} / \omega_{k-1}-2\right) & \left(-\omega_{k} / \omega_{k-1}+2\right) & 0 \\
\left(\omega_{k} / \omega_{k-1}-1\right) & 0 & 0 & \left(2 \omega_{k} / \omega_{k-1}-1\right)
\end{array}\right]
$$

where the ratio $\omega_{k} / \omega_{k-1}$ can be found to be dependent only on the true anomalies before and after the update (see definitions of $\omega$ and $\sigma$ from Eq. 21)

$$
\frac{\omega_{k}}{\omega_{k-1}}=\left(\frac{l \cdot \sigma_{k-1}}{l \cdot \sigma_{k}}\right)^{2}=\left(\frac{1+e \cos \left(v_{k}\right)}{1+e \cos \left(\nu_{k-1}\right)}\right)^{2} .
$$

Therefore, since $l$ does not appear in any other terms of the matrices of Eq. 51, $\boldsymbol{\Psi}$ is not a function of the semi-major axis of the transfer orbit.

On the contrary, by the definition of Eq. 53, B is linearly dependent on $\omega$. This makes $\tilde{\boldsymbol{\Phi}}_{(f, 0)}$ to be a function of the initial and final values of $\omega$ in a 'block-form' as

$$
\tilde{\boldsymbol{\Phi}}_{(f, 0)}=\mathbf{B}^{-1}\left(\omega_{f}\right) \boldsymbol{\Psi}\left(v_{f}, v_{0}\right) \mathbf{B}\left(\omega_{0}\right) \propto\left[\begin{array}{cccc}
1 & 1 & \left(1 / \omega_{f}\right) & \left(1 / \omega_{f}\right) \\
1 & 1 & \left(1 / \omega_{f}\right) & \left(1 / \omega_{f}\right) \\
\omega_{0} & \omega_{0} & 1 & 1 \\
\omega_{0} & \omega_{0} & 1 & 1
\end{array}\right]
$$

For the final solution of Eq. 54, it is necessary to have the complete initial state $\mathbf{p}_{0 \text { tot }}$, but at this stage of the procedure, the part concerning the derivative is not yet known.

A transformation of the primer vector components from the derivative with respect to time, to the derivative with respect to true anomaly (as in Eq. 39) gives

$$
\frac{d p}{d t}=\frac{d p}{d v} \frac{d v}{d t}=\frac{d p}{d \nu} \omega
$$

The product between the primer vector in true anomaly, which is $\mathbf{p}_{t o t, v_{0}}=\left(p_{r 0}, p_{\vartheta 0}, \omega_{0} p_{r 0}^{\prime}\right.$, $\left.\omega_{0} p_{\vartheta 0}^{\prime}\right)^{T}$ and $\tilde{\boldsymbol{\Phi}}_{(f, 0)}$ depends on $\omega$ as

$$
\tilde{\boldsymbol{\Phi}}_{(f, 0)} \cdot \mathbf{p}_{t o t, v_{0}}=\mathbf{p}_{t o t, v_{f}} \propto\left[\begin{array}{cccc}
1 & 1 & \left(\omega_{0} / \omega_{f}\right) & \left(\omega_{0} / \omega_{f}\right) \\
1 & 1 & \left(\omega_{0} / \omega_{f}\right) & \left(\omega_{0} / \omega_{f}\right) \\
\omega_{0} & \omega_{0} & \omega_{0} & \omega_{0} \\
\omega_{0} & \omega_{0} & \omega_{0} & \omega_{0}
\end{array}\right]
$$

Having $\mathbf{p}_{t o t, v_{f}}=\left(p_{r f}, p_{\vartheta f}, \omega_{f} p_{r f}^{\prime}, \omega_{f} p_{\vartheta f}^{\prime}\right)^{T}$, from Eq. 59 clearly $p_{r f}$ and $p_{\vartheta f}$ are independent from the semi-major axis, since the ratio between the initial and final $\omega$ s of Eq. 59 is of the same form of Eq. 56. The last two rows of $\tilde{\boldsymbol{\Phi}}_{(f, 0)} \cdot \mathbf{p}_{t o t, v_{0}}$ of Eq. 59 are linear in $\omega_{0}$. Since they respectively correspond to $\omega_{f} p_{r f}^{\prime}$ and $\omega_{f} p_{\vartheta f}^{\prime}$, this implies that the derivatives in true anomaly of the primer vector are also both independent from $a$.

This is an important conclusion because it states that the primer vector and its derivative are not influenced by the value of the semi-major axis of the transfer orbit. As a result, the size of the search space is reduced and the optimization problem is simplified. 
Fig. 3 Geometric representation of a co-planar departure $\left(e_{D}, a_{D}\right)$, transfer $\left(e_{T}, a_{T}\right)$ and arrival $\left(e_{A}, a_{A}\right)$ orbits case

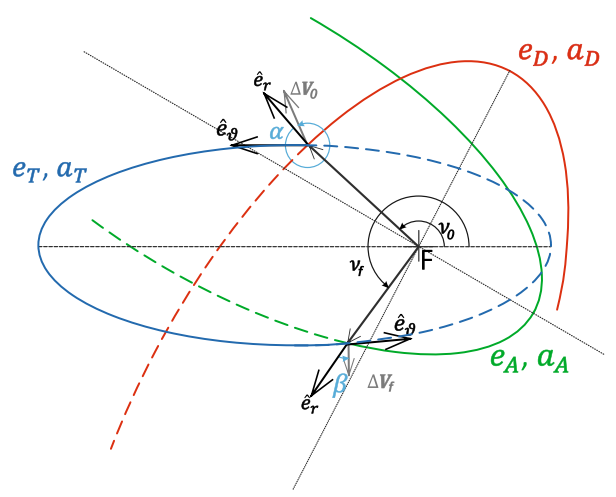

\section{Fixed transfer orbit and variable departure/arrival orbits}

From the definition at the $\mathrm{BC}$ of the primer vector (Eq. 10), $\mathbf{p}_{0}$ and $\mathbf{p}_{f}$ depend only on the directions of the initial and final $\Delta \mathbf{V s}$, but they are independent from their magnitudes. Therefore, a 'natural' application is to consider a problem where the $\Delta \mathbf{V s}$ are parametrized by their directions. In the case where the transfer, departure and arrival orbits are coplanar, the in-plane components of the primer vector can be defined with respect to the orientation of the two local polar reference frames at initial and final position, expressed through two angles $\alpha$ and $\beta$ :

$$
\begin{aligned}
& \mathbf{p}_{0}=\left[\begin{array}{l}
\cos \alpha \\
\sin \alpha
\end{array}\right] \\
& \mathbf{p}_{f}=\left[\begin{array}{l}
\cos \beta \\
\sin \beta
\end{array}\right]
\end{aligned}
$$

If the transfer orbit is fixed (therefore also the velocities $\mathbf{v}_{0}$ and $\mathbf{v}_{f}$ ), the departure and arrival orbits vary according to the orientation (and magnitude) of the $\Delta \mathbf{V s}$ (see Fig. 3).

In the local boundary polar reference frames there is

$$
\begin{gathered}
\mathbf{v}_{0}=v_{r 0} \hat{e}_{r 0}+v_{\vartheta 0} \hat{e}_{\vartheta 0} \\
\mathbf{v}_{f}=v_{r f} \hat{e}_{r f}+v_{\vartheta f} \hat{e}_{\vartheta f}
\end{gathered},
$$

while the $\Delta \mathrm{Vs}$ are

$$
\begin{gathered}
\Delta \mathbf{V}_{0}=\Delta V_{0}\left(\cos \alpha \hat{e}_{r 0}+\sin \alpha \hat{e}_{\vartheta 0}\right) \\
\Delta \mathbf{V}_{f}=\Delta V_{f}\left(\cos \beta \hat{e}_{r f}+\sin \beta \hat{e}_{\vartheta f}\right)
\end{gathered} .
$$

Defining $\mathbf{v}_{D}$ and $\mathbf{v}_{A}$ respectively as the velocity at $\mathbf{r}_{0}$ on the departure orbit and the velocity at $\mathbf{r}_{f}$ on the arrival orbit, they will be

$$
\begin{gathered}
\mathbf{v}_{D}=\mathbf{v}_{0}-\Delta \mathbf{V}_{0}=\left(v_{r 0}-\Delta V_{0} \cos \alpha\right) \hat{e}_{r 0}+\left(v_{\vartheta 0}-\Delta V_{0} \sin \alpha\right) \hat{e}_{\vartheta 0} \\
\mathbf{v}_{A}=\mathbf{v}_{f}+\Delta \mathbf{V}_{f}=\left(v_{r f}+\Delta V_{f} \cos \beta\right) \hat{e}_{r f}+\left(v_{\vartheta f}+\Delta V_{f} \sin \beta\right) \hat{e}_{\vartheta f}
\end{gathered} .
$$

With a fixed transfer orbit, the total state transition matrix $\tilde{\boldsymbol{\Phi}}_{(f, 0)}$ of Eq. 54 is fixed as well. In addition, fixing the transfer orbit means fixing its eccentricity and its semi-major axis. This implies that for this scenario the optimality can vary only if the directions of the $\Delta \mathbf{V s}, \alpha$ and $\beta$, change. In an optimization process, such a problem has a 5 dimensional search space: $e, v_{0}$ and $v_{f}$, which define the transfer orbit, and $\alpha$ and $\beta$, which define the $\mathrm{BC}$ on the $\Delta \mathbf{V}$ s. 


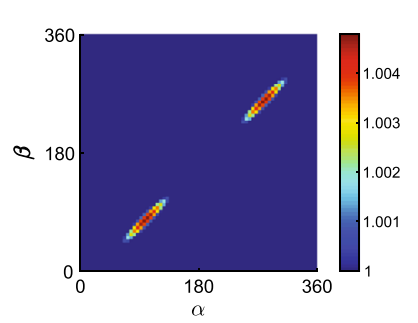

(a) $\nu_{\mathrm{f}}=20.0 \mathrm{deg}$

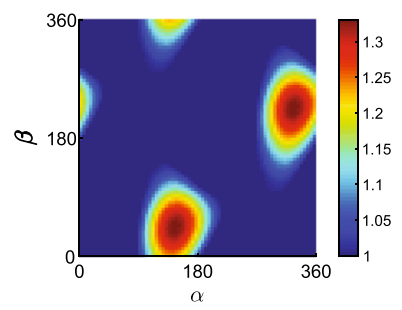

(d) $\nu_{\mathrm{f}}=115.0 \mathrm{deg}$

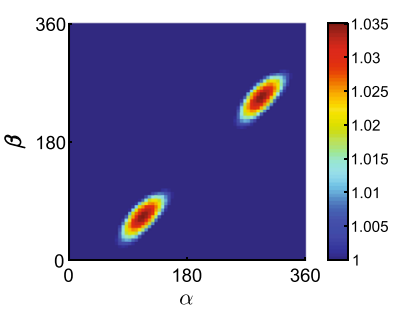

(b) $\nu_{\mathrm{f}}=45.0 \mathrm{deg}$

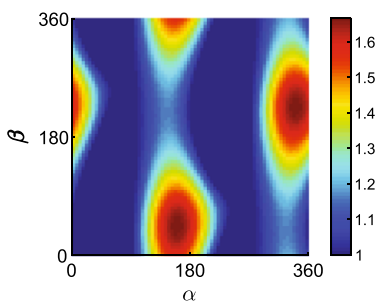

(e) $\nu_{\mathrm{f}}=145.0 \mathrm{deg}$

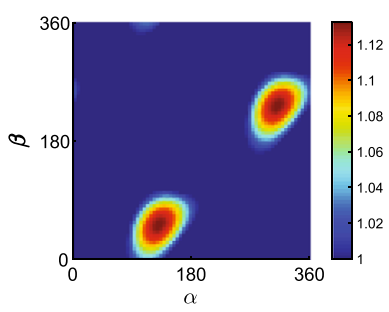

(c) $\nu_{\mathrm{f}}=80.0 \mathrm{deg}$

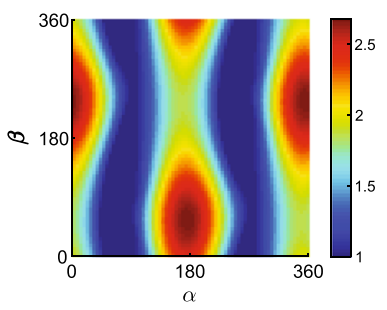

(f) $\nu_{\mathrm{f}}=\mathbf{1 8 0 . 0 \mathrm { deg }}$

Fig. 4 Sequence of maximum values of primer vector maps for a fixed transfer orbit and increasing value of $v_{f}$, versus $\alpha$ and $\beta$. The fixed parameters of the transfer orbit are e $=0.8, \mathrm{a}=0.95 \mathrm{DU}, v_{0}=5 \mathrm{deg}$

Hence, the analysis can be approached differently, having restricted the problem to those 5 dimensions.

Lawden's NCs state that the primer vector is equal to 1 at the $\mathrm{BC}$ and at each intermediate impulse (Lawden 1963). In other words, for a trajectory to be optimal, $p$ must be always smaller than 1 along the arc and equal to 1 at the boundaries, where the $\Delta \mathbf{V s}$ are fired. Within this scenario, a set of transfer orbits with the same eccentricity can be parametrized by, for example, fixing the initial true anomaly and varying $v_{f}$.

Figure 4 illustrates the case where a fixed transfer orbit and fixed $v_{0}$ are considered. The maps show the maximum value of the primer vector according to gradual increasing values of $v_{f}$, for fixed $e=0.8$ and $v_{0}=5 \mathrm{deg}$. They are plotted in a complete interval ( $\left.0-360 \mathrm{deg}\right)$ of $\alpha$ and $\beta$ with a computational step of $5 \mathrm{deg}$.

As already explained, an optimal transfer will have an overall maximum value of the primer vector equal to 1 (dark blues area in Fig. 4). Non-optimal trajectories have $p>1$ and therefore in Fig. 4 they are represented in colours ('non-optimality islands').

The problem is very well structured with a precise geometry: for every specific $v_{f}$ case, the 'non-optimal' islands have always the same dimension, shape and orientation. In particular for small span of true anomalies the 'non-optimal' islands are very stretched but small in size, therefore almost the whole map of Fig. 4a represents an optimal case. There are two main motivations behind this result, one is that for small $\Delta v$, the modulus of the primer vector does not change significantly. In fact, it starts and ends in 1 but its variation along the interval is minimal due to the small span between the initial and final true anomaly. The other reason can be found in the accuracy of the numerical computation $\left(10^{-16}\right)$. Therefore, in Fig. $4 \mathrm{a}$ it can be seen that even 'non-optimal' islands have very low peak values.

Along the different plots shown in Fig. 4, the islands rotate and grow in size and eventually merge for increasingly higher spans of $\Delta v$. The maximum value of the primer vector is always located in the central area of the islands and it increases with an increasing interval of true 


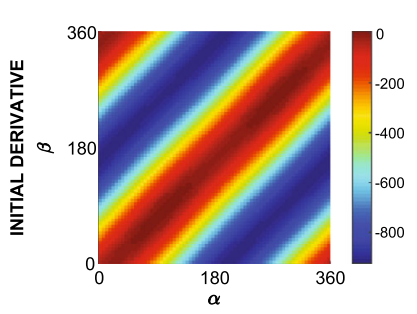

(a) $\nu_{\mathrm{f}}=20.0 \mathrm{deg}$

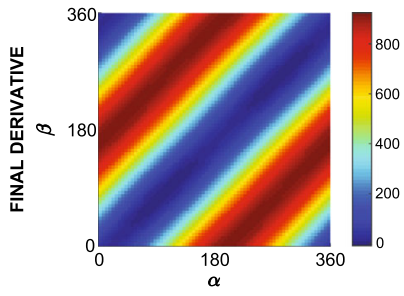

(d) $\nu_{\mathrm{f}}=20.0$ deg

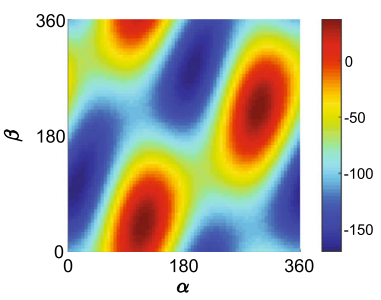

(b) $\nu_{\mathrm{f}}=115.0 \mathrm{deg}$

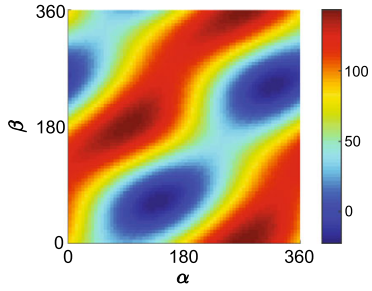

(e) $\nu_{\mathrm{f}}=115.0 \mathrm{deg}$

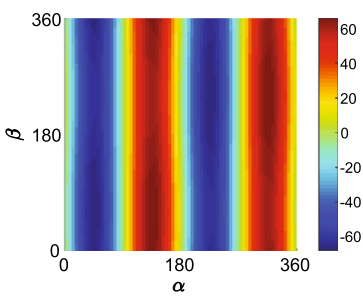

(c) $\nu_{\mathrm{f}}=180.0 \mathrm{deg}$

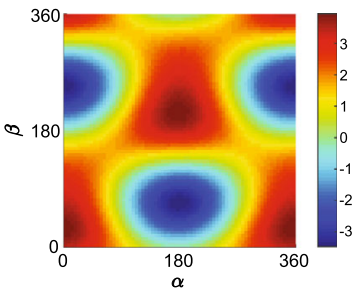

(f) $\nu_{\mathrm{f}}=180.0 \mathrm{deg}$

Fig. 5 Sequence of initial and final derivatives maps for a fixed transfer orbit and increasing value of $v_{f}$, versus $\alpha$ and $\beta$. The characteristics of the transfer orbit are $\mathrm{e}=0.8, \mathrm{a}=0.95 \mathrm{DU}, v_{0}=5 \mathrm{deg}$

anomalies. In fact, while in the case where $\Delta v=15 \mathrm{deg}$ there is $p_{\max } \approx 1$, in the last case $(\Delta v=175 \mathrm{deg}), p_{\max } \approx 3$. These kind of maps allow mission planners not only to visualize the possibility to be in an optimal region but also to change the $\mathrm{BC}$, represented either by the direction of the thrusts ( $\alpha$ and $\beta$ ) or by the true anomalies, to obtain an optimal transfer.

With the same kind of approach, a different scenario can be to exploit the boundary derivatives of the primer vector. It is clear that for an optimal transfer, since the profile of the primer vector has to be smaller than 1 , the initial derivative must be negative while the final derivative must be positive. This condition does not ensure that overall the primer vector will be optimal, but it gives information on the possibility of having an optimal trajectory and on the $\mathrm{BC}$ of the problem. On the other hand, if $\dot{p}_{0}$ is positive and $\dot{p}_{f}$ is negative they definitely define non-optimal trajectories. The expansions at first order of the initial and final derivatives are

$$
\left\{\begin{array}{l}
\dot{p}_{0} \approx \dot{p}_{r 0} p_{r 0}+\dot{p}_{\vartheta 0} p_{\vartheta 0} \\
\dot{p}_{f} \approx \dot{p}_{r f} p_{r f}+\dot{p}_{\vartheta f} p_{\vartheta f}
\end{array},\right.
$$

and they are shown in Fig. 5; specifically, $\dot{p}_{0}$ is represented in Fig. 5a-c, whereas $\dot{p}_{f}$ in Fig. 5d-f. As in Fig. 4, the structure of the maps of Fig. 5 is very well defined. These plots are evaluated (as in the previous case) for fixed values of $e=0.8$ and $v_{0}=5 \mathrm{deg}$, while $v_{f}$ is increasing and $\alpha$ and $\beta$ are defined in the interval from $0 \mathrm{deg}$ to $360 \mathrm{deg}$.

For small true anomaly spans $\left(v_{f}=20.0 \mathrm{deg}\right)$, both the derivatives have a sort of 'stripe' configuration, with opposite values. In fact, it is clear from Fig. $5 \mathrm{a}, \mathrm{d}$ that $\dot{p}_{0}>0$ and $\dot{p}_{f}<0$ and they reach a minimum value of $\approx-800$ and $\approx+800$ respectively. Therefore, the two derivatives define the same boundary optimality areas. On the other hand, for larger $\Delta v, \dot{p}_{0}$ and $\dot{p}_{f}$ tend to smaller absolute values and they both have positive and negative values. Furthermore, as $v_{f}$ increases, the two structures are no longer comparable: they move and stretch tending, in the end, into two completely different scenario. At $v_{f}=180 \mathrm{deg}$, in fact, the initial derivative has a vertical stripes structure (Fig. 5c), while the final derivative has 


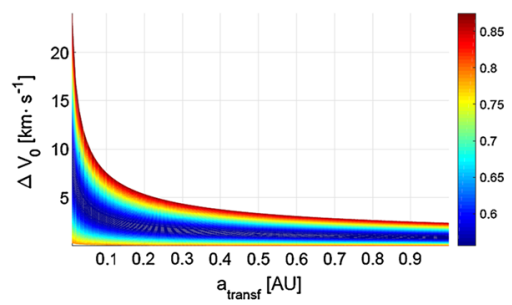

(a)

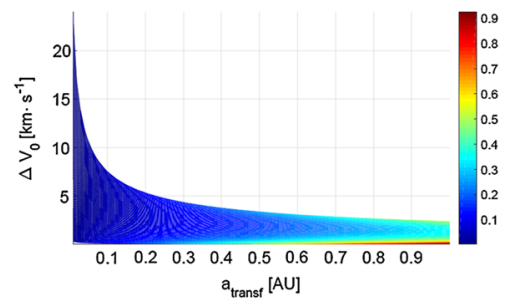

(c)

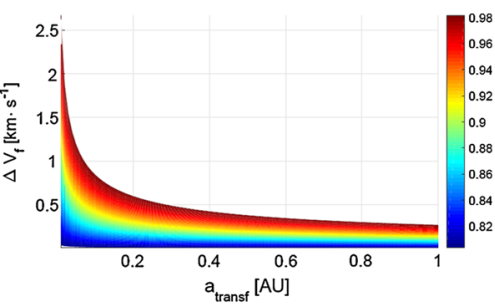

(b)

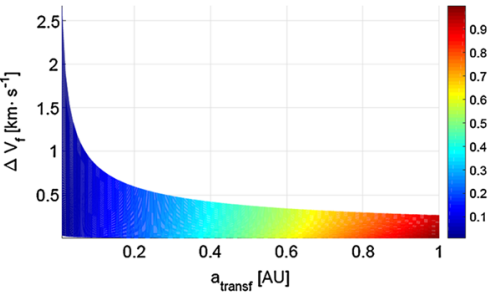

(d)

Fig. 6 Departure (left-side) and arrival (right-side) orbits Keplerian elements (eccentricity and semi-major axis) versus magnitudes of initial and final $\Delta \mathbf{V s}$ and semi-major axis of the transfer orbits

Fig. 7 Primer vector profile, in common to all departure and arrival orbits of Fig. 6

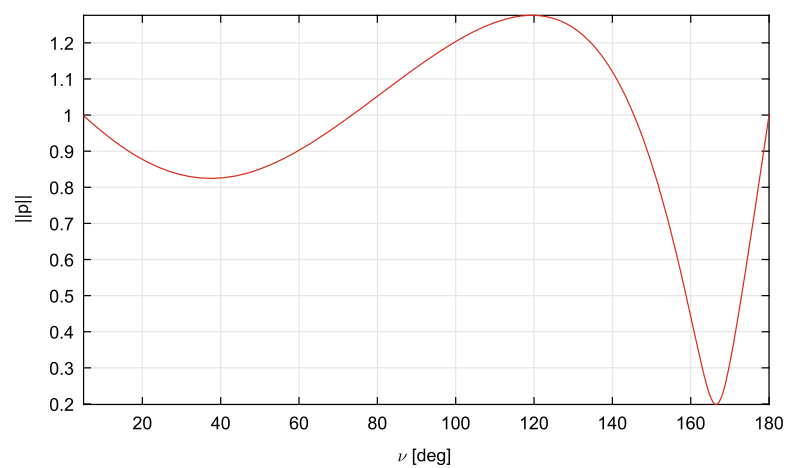

well-defined rounded non-optimal areas (negative value, represented by dark blue and green regions) which have the same size (Fig. 5f).

For both cases (Figs. 4, 5), the most interesting area of the map is represented when the variables cross their critical boundary value (that is 1 for the primer vector and 0 for its derivative). This aspect represents, in fact, the marginal conditions from which a trajectory changes its optimality properties.

The important characteristic of the results presented so far is that they encompass an entire set of orbits with same primer vector profile but different transfer orbits and BC. Specifically, what is varying are the semi-major axis of the transfer orbit $\left(a_{\text {transf }}\right)$ and the magnitudes of the $\Delta \mathbf{V}$ s at the beginning and at the end (therefore the departure and arrival orbits themselves).

An interesting complete scenario is represented in Fig. 6, where it is shown how $e$ and $a$ of the departure and arrival orbits vary according to different magnitudes of $\Delta \mathbf{V}_{0, f}$ and $a_{\text {transf }}$. The fixed parameters are the 5 aforementioned dimensions of the search space: BC in direction of $\Delta \mathbf{V s}$ ( $\alpha$ and $\beta$ ), eccentricity of the transfer orbit and $\mathrm{BC}$ in position $\left(v_{0}\right.$ and $\left.v_{f}\right)$. 
In this specific case the values are $\alpha=30 \mathrm{deg}, \beta=60 \mathrm{deg}, e=0.5, v_{0}=5 \mathrm{deg}$ and $v_{f}=$ $180 \mathrm{deg}$. The simulations have been run with the Sun as central body, a set of semi-major axes of the transfer orbit that goes from $0.01 \mathrm{AU}$ to $1.0 \mathrm{AU}$ and a set of magnitudes of $\Delta \mathbf{V}_{0, f}$ that go from 1 to $80 \%$ of the magnitudes of $\mathbf{v}_{0, f}$ (see Eq. 63).

On the left-hand side of Fig. 7 are the Keplerian elements of the possible departure orbits, while on the right-hand side are the equivalent parameters for the arrival orbits. With the chosen intervals of $a_{\text {transf }}$ and $\Delta \mathrm{V}_{0, f}$, the eccentricities of the departure orbits result in a possible variation from roughly 0.5 to 0.87 , while the ones of the arrival orbits have higher values (0.8-0.96). On the other hand the values of the semi-major axes of the two orbits are roughly comparable, but their variation is different with respect to $a_{\text {transf }}$ and $\Delta \mathbf{V}_{0, f}$.

The main conclusion of this result is that all kinds of departure and arrival orbits summarized in Fig. 6 will meet the same objective, in fact they all correspond to the same primer vector profile (shown in Fig. 7), that in this case is non-optimal.

In summary, this procedure gives a different approach to the primer vector theory and allows one to pursue a complete analysis on entire classes of departure and arrival orbits with common characteristics for specific fixed parameters of the transfer trajectory.

\section{Conclusions}

This paper shows how applying a reference system transformation to the primer vector equation causes the de-coupling between the in-plane and out-of-plane coordinates. The case when the transfer, departure and arrival orbits are co-planar is analyzed. Furthermore, a coordinates transformation is applied with the support of the Hamiltonian of the equations. The Palmer coordinates allow the simplification of the structure of the analysis and give a complete analytical approximation which, given the boundary conditions of the problem, can propagate the primer vector along the arc. For the circular transfer case, Hill's solution is also a solution for the primer vector problem. The primer vector is shown to be independent from the semi-major axis of the transfer orbit and the mathematical complexity and computational time of the problem are reduced. Furthermore, fixing the transfer orbit, it is established that the dimensions of the search space can be reduced to 5. Two approaches to understand the likelihood of the optimality of a specific set of transfers are examined in the paper, with related optimality maps. Finally it is shown that a single profile of primer vector is related to a set of departure and arrival orbits through the directions of the initial and final $\Delta \mathbf{V}$ s and the semi-major axis of the transfer trajectory. The simplicity of the new procedure allows the exploitation of many other properties of the problem, not presented in this paper.

Acknowledgments This research has been funded by the European Commission through the Marie Curie Initial Training Network PITN-GA-2011-289240, AstroNet-II The Astrodynamics Network.

Open Access This article is distributed under the terms of the Creative Commons Attribution 4.0 International License (http://creativecommons.org/licenses/by/4.0/), which permits unrestricted use, distribution, and reproduction in any medium, provided you give appropriate credit to the original author(s) and the source, provide a link to the Creative Commons license, and indicate if changes were made.

\section{References}

Battin, R.H.: An Introduction to the Mathematics and Methods of Astrodynamics, Revised edn. AIAA Education Series (1999) 
Bernelli-Zazzera, F., Lavagna, M., Armellin, R., Di Lizia, P., Topputo, F., Berz, M., et al.: Global Trajectory Optimization: Can We Prune the Solution Space When Considering Deep Space Maneuvers? In: Technical report, ESA (2007)

Bryson, A.E., Ho, Y.: Applied Optimal Control, Optimization, Estimation, and Control. Taylor \& Francis, London (1975)

Conway, B.A. (ed.): Spacecraft Trajectory Optimization. Cambridge University Press, Cambridge (2010)

Goodyear, W.H.: Completely general closed-form solution for coordinates and partial derivatives of the twobody problem. Astron. J. 70(3), 189-192 (1965)

Jezewski, D.J.: Primer Vector Theory And Applications. In: Technical report, Lyndon B. Johnson Space Center, NASA (1975)

Jezewski, D.J., Rozendaal, H.L.: An efficient method for calculating optimal free-space n-impulse trajectories. AIAA J. 6(11), 2160-2165 (1968)

Lawden, D.F.: Optimal Trajectories for Space Navigation. Butterworths Mathematical Texts, London (1963)

Lion, P.M., Handelsman, M.: Primer vector on fixed-time impulsive trajectories. AIAA J. 6(1), 127-132 (1968)

Luo, Y., Zhang, J., Li, H., Tang, G.: Interactive optimization approach for optimal impulsive rendezvous using primer vector and evolutionary algorithms. Acta Astronaut. 67, 396-405 (2010)

Olympio, J.T., Marmorat, J.P., Izzo, D.: Global Trajectory Optimization: Can We Prune the Solution Space When Considering Deep Space Maneuvers?. In: Technical report, ESA (2006)

Palmer, P.L.: Reachability and optimal phasing for reconfiguration in near circular orbit formations. J. Guid. Control Dyn. 30(5), 1542-1546 (2007)

Prussing, J.E.: Optimal impulsive linear systems: sufficient conditions and maximum number of impulses. J. Astronaut. Sci. 48(2), 195-206 (1995)

Quinn, T., Perrine, R.P., Richardson, D.C., Barnes, R.: A symplectic integrator for Hill's equations. Astron. J. 139, 803-807 (2010)

Vasile, M., De Pascale, P.: Preliminary design of multiple gravity-assist trajectories. J. Spacecr. Rockets 43(4), 794-805 (2006)

Vinh, N.X.: Integration of the primer vector in a central force field. J. Optim. Theory Appl. 9(1), 51-58 (1972) 